\title{
Management of hypertension with biofeedback in caregiver burden: Monitoring and control of hypertension
}

\author{
Manejo de la hipertensión con biofeedback en la carga del cuidador: seguimiento y \\ control de la hipertensión
}

\author{
Antonio Aguilar-Agudo ${ }^{1,2 *}$ \\ ${ }^{1}$ Asociación Parkinson Córdoba, Faculty of Educational Sciences; ${ }^{2}$ Department of Psychology, Risk Behavior, Health and Occupational Safety \\ Research Group, Faculty of Educational Sciences. Universidad de Córdoba. Córdoba, Spain
}

The case is presented of a 59 -year-old woman who was the caregiver of her husband, who had Parkinson's disease and dementia. She attended due to caregiver burden-related problems. She had symptoms of psychosocial stress, headaches, insomnia, and high blood pressure (BP). He had increased tobacco consumption, which was so far moderate. She was referred for medical evaluation, with baseline BP normalization being achieved (Fig. 1) after receiving pharmacological treatment (ramipril, $5 \mathrm{mg}, 1$ tablet/day) for hypertension $(\mathrm{HT})$. However, BP increases continued to be common in scenarios experienced as stressful during the care of her spouse. This was putting the patient's health at risk and was an obstacle to continue playing her role as caregiver.

The Joint National Committee $7^{1}$ (JNC-7) proposes adopting healthy lifestyles for HT control. For the JNC$8^{2}$, care-related decisions should consider and incorporate clinical characteristics and circumstances of each individual patient, and reflect that physicians want guidance on the management of HT with the best scientific evidence available. The American College of Cardiology and the American Heart Association guidelines ${ }^{3}$ establish the relationship between HT and psychosocial stress, characterizing it as a relatively fixed risk factor (RF). Several publications refer to the effect of non-pharmacological interventions, by means of the use of transcendental meditation techniques, on $\mathrm{HT}$, with the methodological weaknesses of this type of treatments standing out ${ }^{4}$. The 2017 guidelines emphasize a systolic pressure $<130 \mathrm{mmHg}$ and diastolic pressure $>80 \mathrm{mmHg}$ as therapeutic goal.

In this sense, this document describes a non-pharmacological treatment for the management of HT (not associated with organic problems) and thereby for the elimination of a RF for cardiovascular (CV) diseases. The focus is on the "caring for the caregiver" strategy. The central question when assessing this method is to determine how the study arrived to its conclusions, with the purpose to facilitate clear communication of research findings to stimulate the publication of relevant investigations.

Numerous publications show evidence for considering biofeedback (BFB) treatment as an alternative that increases awareness of maintaining control over BP, encouraging the adoption of a healthy lifestyle ${ }^{5}$. In addition, it offers objective and measurable data. The 2016 ESC guidelines ${ }^{6}$ propose helping people understand the relationship between their behavior and health as a strategy to facilitate behavioral change.

\section{Correspondence:}

*Antonio Aguilar-Agudo 


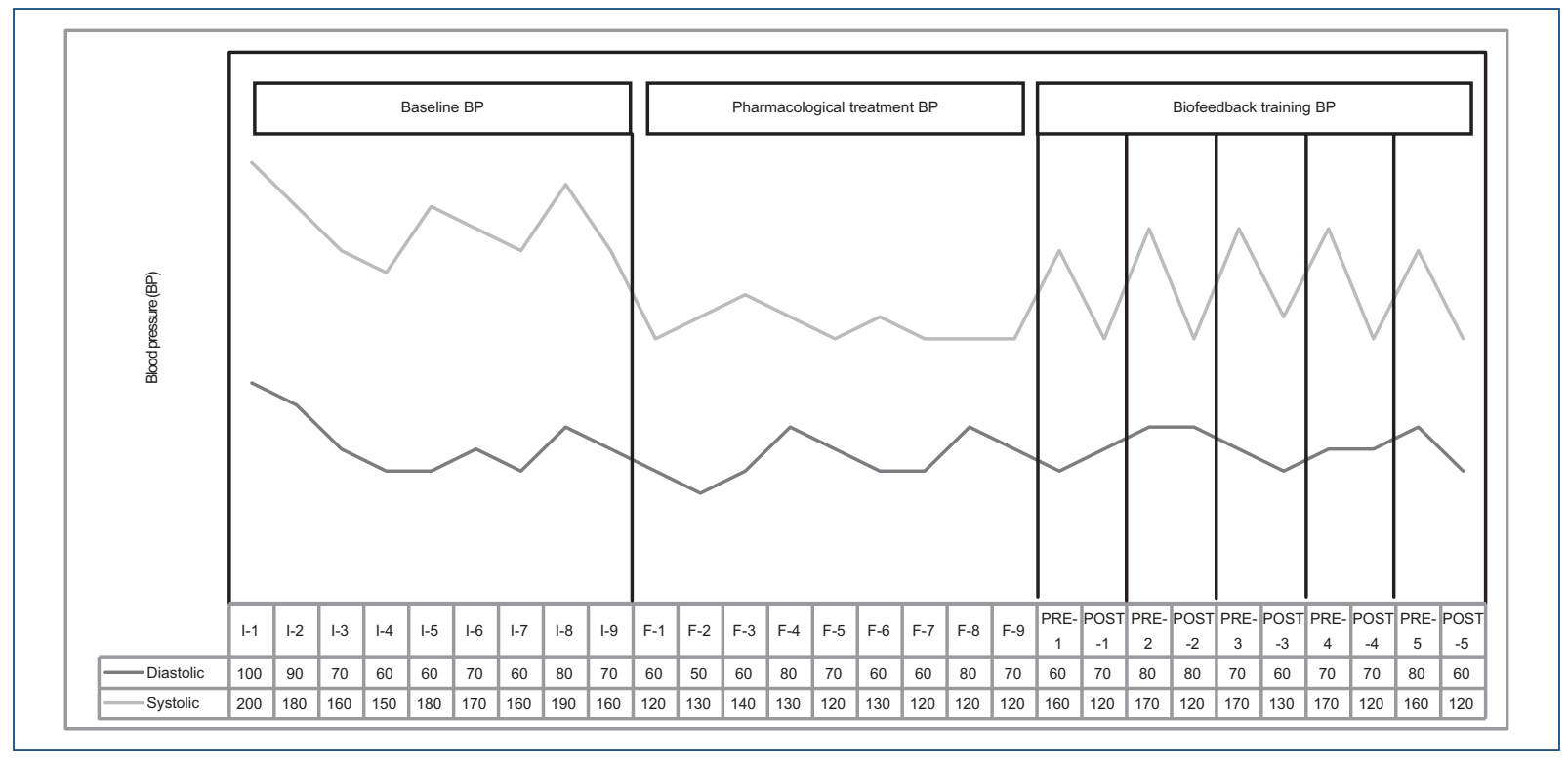

Figure 1. Blood pressure records: at baseline $(B)$; after pharmacological treatment $(P)$; during five real stressful situations with BP measurements before (pre) and after (post) practicing abdominal breathing at home.

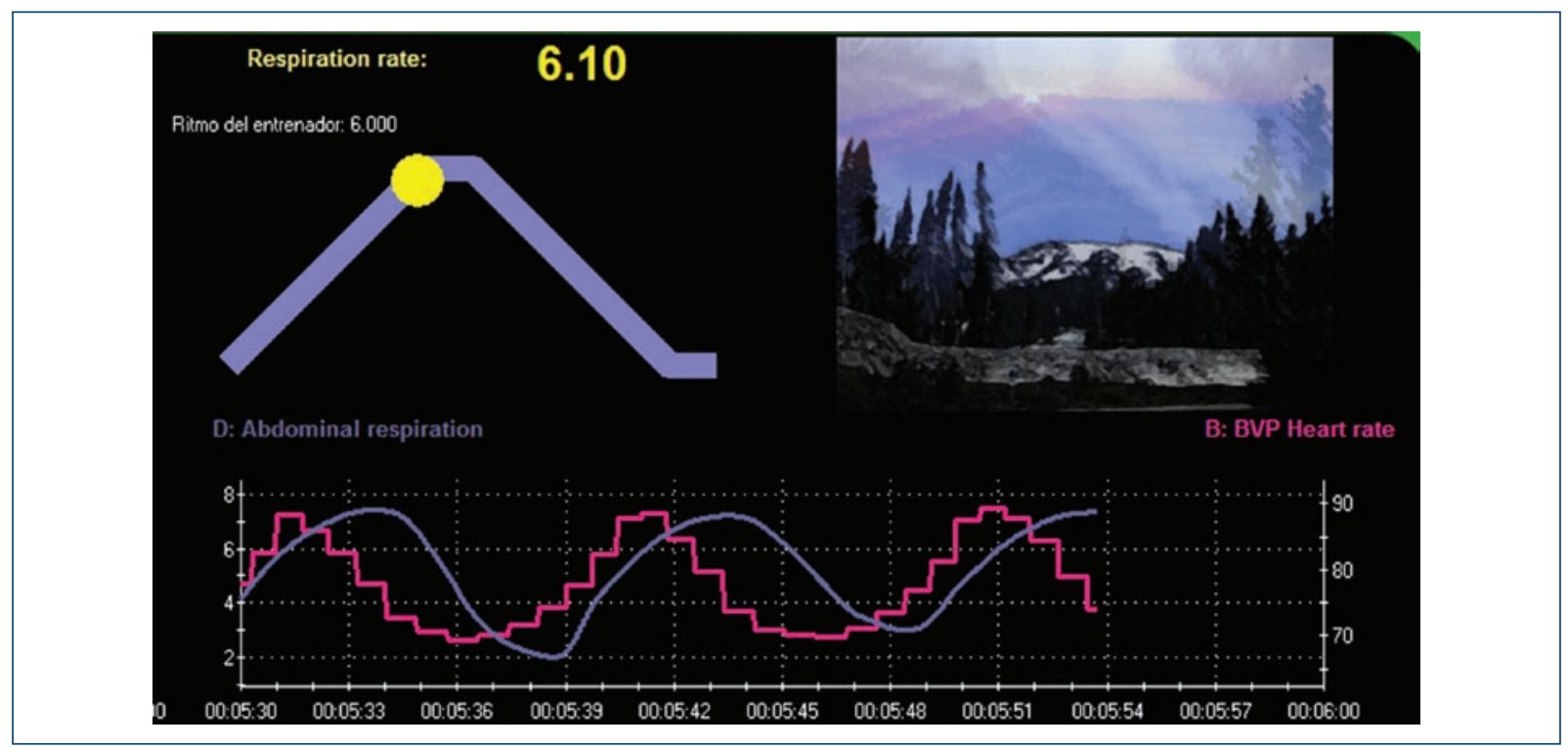

Figure 2. Visual signals (RESP and BVP) provided to the patient during the intervention phase (on top, trainer for abdominal breathing exercises). Ritmo del entrenador. trainer rhythm. (Source: researcher's own).

Comments on the guidelines ${ }^{7}$ include contributions regarding individual intervention on RFs: engaging the patient in the management of his/her disease and the recommendation to intervene if the psychosocial RF is diagnosable or if it can contribute to worsen CV diseases stand out as strategies.

During the study, BFB training was carried out using the ProComp5 ${ }^{T M}$-Infinit ${ }^{\circledR}$ system (Thought Technology Ltd.), using the BVP (pulse volume) and RESP (respiratory rate, RR) sensors. During each session, visual signals were provided on a computer screen: RESP, which could measure abdominal frequency and amplitude at each breath, and in combination with BVP, it provides heart rate variability (HRV) monitoring. A 6-breath/min protocol was used for training, as a pattern to converge on an optimal HRV. Ten 20-min sessions were held over 5 weeks. The practice of abdominal breathing $(A B)$ exercises at home was 
proposed. After the training phase, the patient had acquired the skills to synchronize her HR with her RR (Fig. 2) and the skills for the execution of a practical and simple technique, without the need to receive the visual signals.

Some trials were carried out with the patient, and $A B$ use was proposed after perceiving signs of tension and anxiety in everyday stressful situations. The review of BP records (Fig. 1) indicated a greater ability in the patient to show a relaxed behavior in stressful situations after $A B$, thereby causing a $B P$ reduction. During the entire process, the patient was instructed not to interrupt the pharmacological treatment, with any decision on the subject corresponding to her reference doctor, who should regularly monitor BP control.

This study describes how a patient acquired skills (learned breathing pattern) for the management of high BP associated with the care of her spouse. The reduction of the symptoms caused by caregiver burden through the development of skills for self-regulation can have an impact on caregivers' quality of life and, as a consequence, also on people who are the recipients of care.

\section{Funding}

The present investigation has not received specific aid from public or commercial sector agencies or non-profit entities.

\section{Conflicts of interest}

The author declares that they have no conflicts of interest.

\section{Ethical disclosures}

Protection of people and animals. The author declares that the procedures that were followed adhered to the ethical standards of the responsible committee on experimentation in human subjects and were in accordance with the World Medical Association and the Declaration of Helsinki.

Confidentiality of data. The author declares that he has followed the protocols of his work center on the publication of patient data.

Right to privacy and informed consent. The author has obtained informed consent of the patients and/or subjects referred to in the article. This document is in the possession of the author.

\section{References}

1. Chobanian AV, Bakris GL, Black HR, Cushman WC, Green LA, Izzo JL Jr, et al.; Joint National Committee on Prevention, Detection, Evaluation, and Treatment of High Blood Pressure. National Heart, Lung, and Blood Institute; National High Blood Pressure Education Program Coordinating Committee. Seventh Report of the Joint National Committee on Prevention, Detection, Evaluation, and Treatment of High Blood Pressure. Hypertens. 2003;42:1206-52.

2. James PA, Oparil S, Carter BL, Cushman WC, Dennison-Himmelfarb C, Handler J, et al. 2014 Evidence-based guideline for the management of high blood pressure in adults: Report from the Panel Members Appointed to the Eighth Joint National Committee (JNC 8). JAMA. 2014;311(5):507-20.

3. Whelton PK, Carey RM, Aronow WS, Casey DE Jr, Collins KJ, Dennison Himmelfarb C, et al. 2017 ACC/AHA/AAPA/ABC/ACPM/AGS/APhA/ASH/ ASPC/NMA/PCNA Guideline for the Prevention, Detection, Evaluation, and Management of High Blood Pressure in Adults: Executive Summary: A Report of the American College of Cardiology/American Heart Association Task Force on Clinical Practice Guidelines. Hypertens. 2018;71:1269-324.

4. Canter PH, Ernst E. Insufficient evidence to conclude whether or not Transcendental Meditation decreases blood pressure: results of a systematic review of randomized clinical trials. J Hypertens. 2004;22(11):2049-54.

5. Nakao M, Yano E, Nomura S, Kuboki T. Blood pressure-lowering effects of biofeedback treatment in hypertension: a meta-analysis of randomized controlled trials. Hypertens Res. 2003:26:37-46.

6. Arrarte V, Berenguel A, Gonzalez C, de la Hera JM, Lekuona I, Marzal D, et al. Guía ESC-2016 sobre prevención de la enfermedad cardiovascular en la práctica clínica. Rev Esp Cardiol. 2016:69(10):939.

7. Arrarte V, Berenguel A, Gonzalez C, de la Hera JM, Lekuona I, Marzal D, et al. Comentarios a la guía ESC-2016 sobre prevención de la enfermedad cardiovascular en la práctica clínica. Rev Esp Cardiol. 2016;69(10):894-9. 